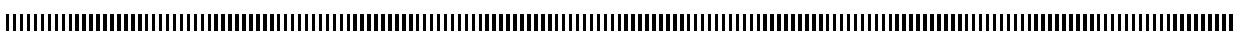

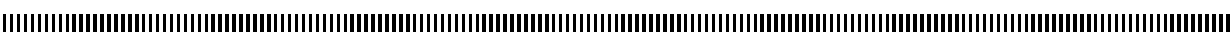

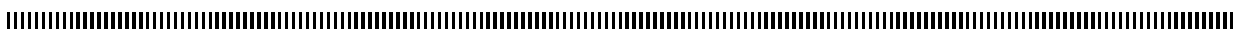

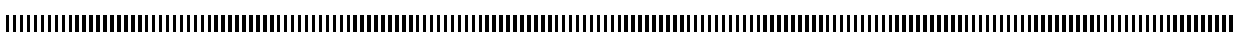

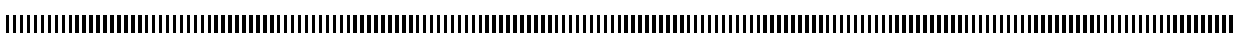
| | | | |

\title{
Migrations in the Rosenzweig-MacArthur model and the "atto-fox" problem
}

\author{
Claude Lobry $^{*}$ - Tewfik Sari ${ }^{* *}$ \\ (*) Modemic \\ INRIA et UNSA \\ Le Gd Palais, 2 Bv de Cimez, 06000 Nice \\ France \\ claude.lobry@inria.fr \\ (**) Irstea UMR ITAP \\ 361 rue Jean-François Breton \\ 34196 Montpellier Cedex \\ France \\ tewfik.sari@irstea.fr
}

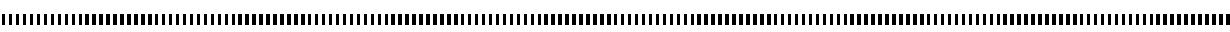

ABSTRACT. The Rosenzweig-MacArthur model is a system of two ODEs used in population dynamics to modelize the predator-prey relationship. For certain values of the parameters the differential system exhibits a unique stable limit cycle. When the dynamics of the prey is faster than the dynamics of the predator, during oscillations along the limit cycle, the density of preys take so small values that it cannot modelize any actual population. This phenomenon is known as the "atto-fox" problem. In this paper we assume that the populations are living in two patches and are able to migrate from one patch to another. We give conditions for which the migration can prevent the density of prey being too small.

RÉSUMÉ. Le modèle de Rosenzweig-MacArthur est un système de deux équations différentielles utilisé en dynamique des populations pour modéliser la relation entre un prédateur et sa proie. Pour certaines valeurs des paramètres le système possède un cycle limite unique stable. Lorsque la dynamique de la proie est plus rapide que celle du prédateur durant les oscillations le long du cycle, la densité des proies atteint des valeurs tellement petites qu'elle ne peut modéliser une situation issue du monde réel. Ce phénomène est connu sous le nom du problème "Atto-Fox". Dans cet article on suppose que les populations sont réparties sur deux sites et qu'elles peuvent migrer de l'un à l'autre. Nous donnons des conditions qui assurent que la migration va empêcher la densité des proies de devenir trop petite.

KEYWORDS : Rosenzweig-MacArthur model, migration, atto-fox problem, singular perturbations, limit cycles, nonstandard analysis, canards.

MOTS-CLÉS : Modèle de Rosenzweig-MacArthur, migration, problème atto-fox, perturbations singulières, cycles limites, analyse non standard, canards.

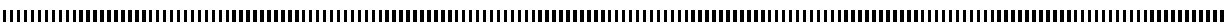




\section{Introduction}

This paper is dedicated to E. Benoît. His pioneering work [3] with J-L. Callot, F. Diener and M. Diener is our source of inspiration. We know that he is presently working on highly sophisticated problems on "canards" when time is complex and we have no doubt that he will obtain great achievements in this direction. Nevertheless we hope that the present paper will comfort him in the idea that the simple and elegant arguments of NSA which were very successful in the theory of slow-fast systems in $\mathbb{R}^{2}$ and $\mathbb{R}^{3}$ are still useful for some more complex systems.

The Rosenzweig-MacArthur model (RMA model) is the following system of two differential equations widely used in population dynamics to modelize the predator-prey (or resource-consumer) relationship (see [2], Ch. 1-2 pp 14-17).

$$
\left\{\begin{array}{l}
\frac{d x}{d t}=x g(x)-h(x) y \\
\frac{d y}{d t}=\varepsilon h(x) y-\delta y
\end{array}\right.
$$

where :

1) $x \mapsto g(x) ; x \geq 0$ is continuous, decreasing and equal to 0 for some value $K$.

2) $x \mapsto h(x) ; x \geq 0$ is continuous, increasing, null for $x=0$ and bounded.

3) $\delta$ is a positive constant.

4) $\varepsilon>0$ is a small parameter.

Rosenzweig-MacArthur model refers more precisely to the case where $x \mapsto x g(x)$ and $x \mapsto h(x)$ are respectively a logistic and a Monod's function.

This is certainly not the best way to represent trophic interaction. As explained in [2], in the ecological theory, it should be better to use in place of the function $x \mapsto h(x)$ a function of the two variables $x$ and $y:(x, y) \mapsto h(x, y)$, which is still increasing with respect to $x$ but decreasing with respect to $y$. We shall not do that because we want to concentrate on the mathematics of the model rather than on the ecological interpretation, and the mathematics are simpler with $h(x)$ in place of $h(x, y)$. Moreover, as we shall explain later, taking the more general $h(x, y)$ does not change much what we want to explore here.

It is known (see [2,9]) that for certain values of the parameters the system (1) exhibits a unique limit cycle. When $\varepsilon$ is small the minimum of the value of $x(t)$ along the limit cycle is exponentially small with respect to $\varepsilon$, so small that it cannot modelize any actual population (see [7, 16]). After Mollisson [16] (see also [11] on the same question) we call

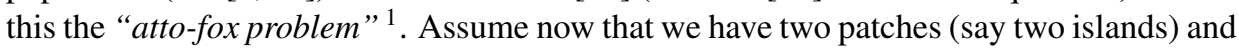

1. This comes from the fact that in a specific model a variable representing the number of foxes per square kilometer decreases to $10^{-18}$ before it re-increases. 
that populations are able to migrate from one patch to another. Intuitively we understand that migration can prevent $x(t)$ of being too small if the two populations associated to $x$ on each patch are not small at the same time, the migration from the large population preventing the small one to become extinct. Our objective is to make more precise on a mathematical model this vague statement. Our model resorts to what is called coupled oscillators and is widely considered in various areas like electronics, mechanical systems, neurosciences, etc. We do not know whether our model was considered sometimes in those area with the same aims but, in theoretical ecology, our questioning seems to be new.

In the first section we recall already known facts about the Rosenzweig - MacArthur model in the case where $\varepsilon$ is small. For this purpose we shall use the language of Non Standard Analysis I.S.T. introduced by E. Nelson in [20] and popularized by E. Benoît, J-L. Callot, F. Diener and M. Diener in [3] in the study of slow-fast systems ; the reader who is not familiar with these technics can consider the intuitive meaning of the words. The interested reader will find an introduction to this way of thinking in [10] or [15]. As we did in [15] and a previous paper on the RMA model [16] we shall use bold letters for terms like $a$ is infinitesimal which has a formal interpretation in I.S.T. :

$$
a \text { is infinitesimal } \Longleftrightarrow\left\{\forall^{s t} t>0 \quad|a| \leq t\right\}
$$

The second section is devoted to our model of coupled oscillators. We will present the model, show simulations and sketch the proof of a non classical result. By non classical we mean that the result is stated in the language of I.S.T. and that we did not try to find a classical equivalent. Whether this result answers correctly the atto-fox question is discussed in the conclusion.

The presence of an "atto-fox problem" in the RMA model was quoted by us in [16]. The question of migration between two patches was considered in the unpublished paper [8].

\section{Classical results about the Rosenzweig-MacArthur model}

Since we intend to present a lot of simulations, we must specify the functions $g$ an $h$ of (1). For that purpose we consider :

$$
\left\{\begin{aligned}
\frac{d x}{d t} & =2 x(1-x)-\frac{x}{0.1+x} y \\
\frac{d y}{d t} & =\varepsilon \frac{x}{0.1+x}-\delta y
\end{aligned}\right.
$$

The choice of 2 in the expression $2 x(1-x)$ is just for esthetic reason, the parabola defining the x-isocline being less "flat" than the one with 1 in place of 2 . We assume that $\varepsilon$ is infinitely small and that $\delta=\varepsilon m$ with $m$ limited. If $\delta$ was not infinitesimal but 
appreciable, $y(t)$ would be all the time decreasing and the model of poor interest. Thus our system is a slow-fast differential system that we prefer to reconsider, after a change of unit of time, as :

$$
\left\{\begin{aligned}
\frac{d x}{d t} & =\frac{1}{\varepsilon}\left(2 x(1-x)-\frac{x}{0.1+x} y\right) \\
\frac{d y}{d t} & =\left(\frac{x}{0.1+x}-m\right) y
\end{aligned}\right.
$$

\subsection{The RMA-model without migration}

We recall some very well known properties of the system (3). The positive orthant $\left(\mathbb{R}^{+}\right)^{2}=\{(x, y): x \geq 0 ; y \geq 0\}$ is invariant, all solutions are bounded and, by the way, defined for all positive $t_{s}^{\prime}$. We are only interested by solutions in the positive orthant. The open positive orthant $\left(\mathbb{R}^{*+}\right)^{2}=\{(x, y): x>0 ; y>0\}$ is also invariant.

\section{Notations}

The $x$-isocline is the union of the two arcs of parabola :

$$
\mathcal{P}^{+}=\{(x, y): 0.45 \leq x \leq 1 ; y=2(1-x)(0.1+x)\}
$$

and

$$
\mathcal{P}^{-}=\{(x, y): 0 \leq x \leq 0.45 ; y=2(1-x)(0.1+x)\}
$$

and the two parts of the $y$ axis :

$$
\Pi^{+}=\{(x, y): x=0 ; 0.2<y\}
$$

and

$$
\Pi^{-}=\{(x, y): x=0 ; 0<y<0.2\}
$$

The $y$-isocline is the union of the $x$-axis and the vertical segment :

$$
\Pi_{m}=\left\{(x, y): x=\frac{0.1 m}{1-m}, y>0\right\}
$$

See Fig. 1.

\section{Non Standard Notations}

$-\varepsilon$ is a fixed strictly positive infinitesimal. The function $g, h$ in (1) and the parameter $m$ in (3) are standard.

- The $x$-isocline is also the slow curve of (3). This slow curve is divided in two parts :

- The attracting one : $\Pi^{+} \cup \mathcal{P}^{+}$ 


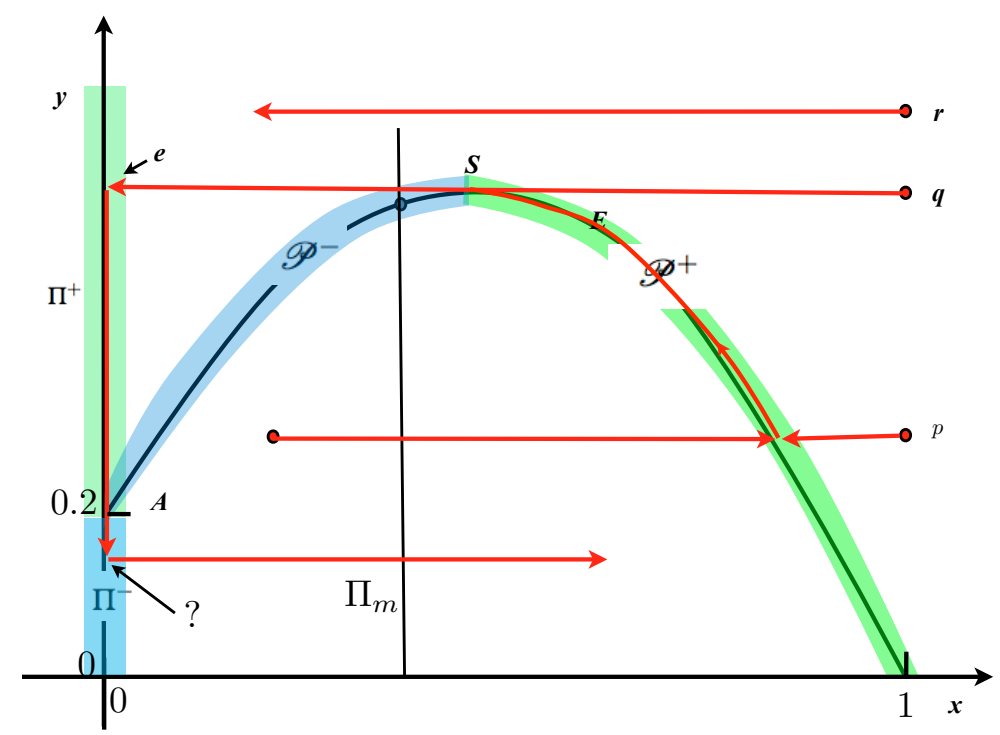

Figure 1. Scheme of the slow-fast system (3)

- The repelling one : $\Pi^{-} \cup \mathcal{P}^{-}$

- The (external) set of points which are at an infinitesimal distance of some set $M$ is called the halo of $M$ and denoted by $\operatorname{hal}(M)$. Since $\mathbf{h a l}(M)$ is an external set the expression $(x, y) \in \mathbf{h a l}(M)$ is a misuse which means that the distance of $(x, y)$ to $M$ is infinitesimal.

\section{Phase portrait : Limit cycle case.}

This is the case when $\Pi_{m}$ is on the left of $S$ and not infinitely close to it. On Fig.1 one observes the classical scheme for solutions of (3) when $\varepsilon$ is infinitely small. The solution starting from point $p$ is "quasi-horizontal" and enters the halo (in green) of the stable part of the slow curve $\mathcal{P}^{+}$; then the solution will remain in $\mathbf{h a l}\left(\mathcal{P}^{+}\right)$, moving up (because we are on the right of $\Pi_{m}$ until it reaches the summit $S$ where it jumps to the halo of the other stable part of the slow curve $\Pi^{+}$at the point $e$ ( $e$ for "entrance" or "entrée" in french)) ; then the solution will decrease (since $\Pi^{+}$is on the left of $\Pi_{m}$ ) following the stable part of the slow curve and penetrates into the halo of the unstable part $\Pi^{-}$of the slow curve (in blue) ; the solution will leave the slow curve at some point ; for the moment we have no information regarding the exact position of this exit point ; the entrance-exit relationship is the object of the next subsection.

The existence of a limit cycle is straightforward when $\varepsilon \approx 0$. Consider the Poincaré return map $\varphi$ defined on a segment of the form :

$$
\Delta=\{(x, y): x=0.45 ; y \in[0.5,0.7]\}
$$


Any solution starting from a point $(0.45, y)$ of $\Delta$ will cross $\Delta$ after one turn at a point $(0.45, \varphi(y))$ such that $\varphi(y) \approx 0.605$; thus $\varphi(y)$ maps $\Delta$ into $\Delta$ and by the intermediate value theorem there exist at least one fixed point. Unicity is a bit more delicate and we refer to [9] for a proof.

\section{Simulation : Limit cycle case.}

All the simulations are done using an Euler scheme with step $d t:=10^{-4}$. It might be surprising to use such a primitive integration routine. We have some reasons for this:

1) For such systems, duration of integration does not matter. It is quasi instantaneous.

2) Good agreement between simulations and theoretical results when available is observed.

3) The model (3) is justified on the basis of an Euler scheme.

The simulation of Fig. 2 is performed with $\varepsilon=0.05$ and $m=0.7$. One sees the unstable equilibrium $E$ with trajectories issued from points in its neighbourhood (actually they are simulated using reversed time) and we see all the red trajectories converging to the limit cycle in blue.

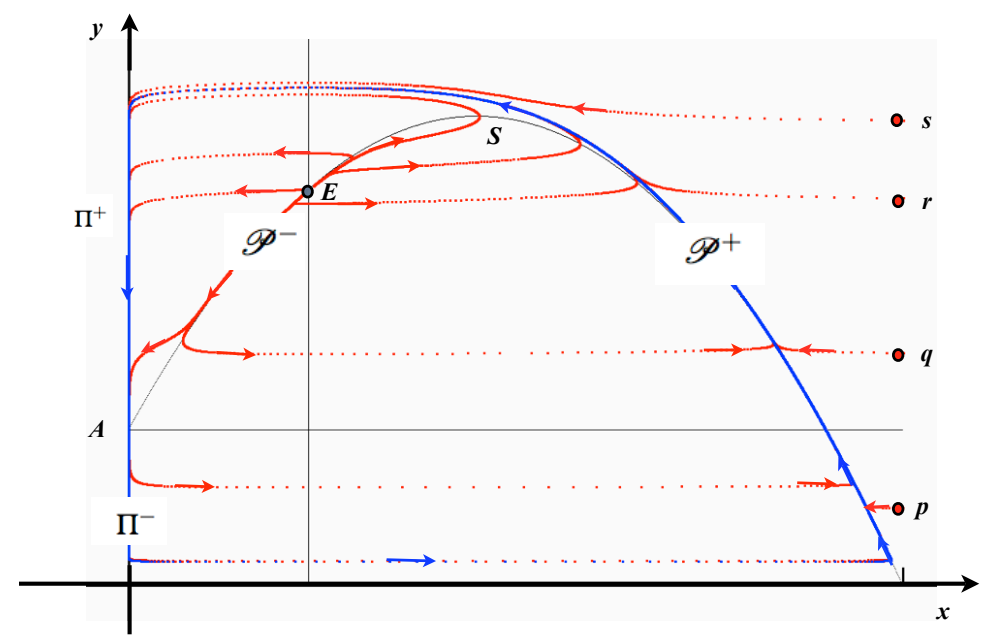

Figure 2. Phase portrait of (3) $: \varepsilon=0.05, m: 0.7$

On Fig. 3 we have just represented the limit cycle with numerical values for some characteristic points. On Fig. 4 one sees the graphs of the function $t \mapsto x(t)$ (in red) 


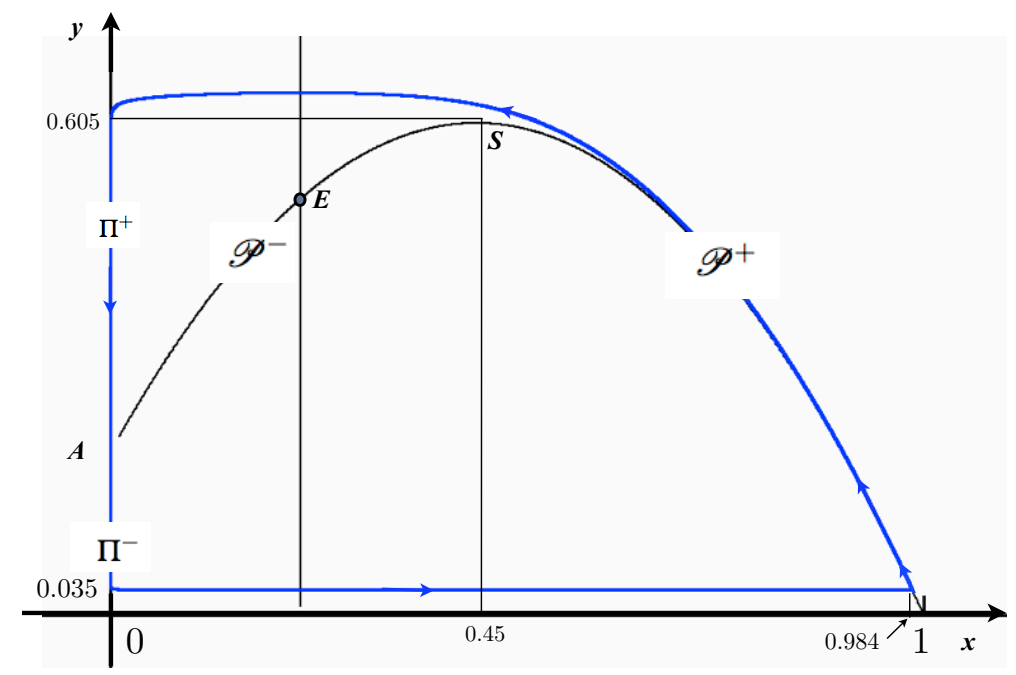

Figure 3. Limit cycle of (3) $: \varepsilon=0.05, m: 0.7$

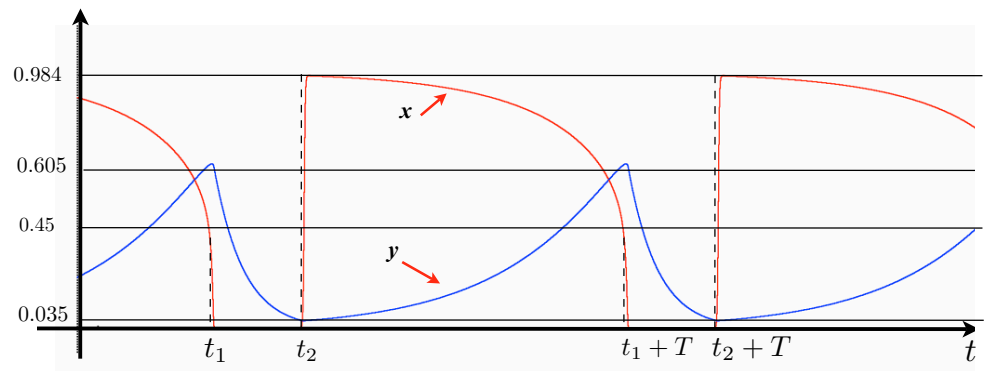

Figure 4. Limit cycle of (3); "jumps" of $x(t): \varepsilon=0.05, m: 0.7$

and the function $t \mapsto y(t)$ (in blue). The function $t \mapsto x(t)$ is not $\mathbf{S}$-continuous ${ }^{2}$; the simulation presents "jumps" at times $t_{1}, t_{1}+T$ (where $T$ is the period of the limit cycle) from 0.45 to 0 and at times $t_{2}, t_{2}+T$ from 0 to 0.984 ; actually the value 0 is not attained by the "true" solution but is infinitesimal. In both cases the "duration" of the jump is

2. The (nonstandard) function $f$ is S-continuous if

$$
\forall x \quad d x \approx 0 \Longrightarrow f(x+d x) \approx f(x)
$$


infinitesimal. Here "jump" and "duration" are not formally defined. It will be done using the notion of 'shadow' just at the end of the next section.

As we just said the true value of the minimum attained by $x$ is not 0 . If we ask to the computer what is the value of the minimum we obtain something smaller than $10^{-20}$ ! One question rises : is this a property of the system or a pure numeric artifacts ? Since in some places we will use numerical simulations in place of mathematical arguments we must clarify this point. We also will do it in the next section.

\subsection{The "entrance-exit" relationship}

Consider an initial condition $\left(x_{0}, y_{0}\right)$ which is in the halo of $\Pi^{+}$and such that $x_{0}>0$. We have seen that this solution will "re-appear", i.e. will leave the halo of $\Pi$ at some point which depends only on $y_{0}$. More precisely we have the proposition :

Proposition 2.1 Assume that the initial condition $\left(x_{0}, y_{0}\right)$ in hal $\left(\Pi^{-}\right)$is such that:

$-y_{0}>0.2$ and not infinitely large

$-\xi_{0}=\varepsilon \log \left(x_{0}\right) \approx 0$

Let the number $t_{1}$ defined by:

$$
\int_{t_{0}}^{t_{1}}\left(2-10 y_{0} \exp \left(-m\left(s-t_{0}\right)\right)\right) d s=0
$$

then the solution $\left(x\left(t, t_{0},\left(x_{0}, y_{0}\right)\right), y\left(t, t_{0},\left(x_{0}, y_{0}\right)\right)\right)$ of (3) has the property:

$$
\begin{aligned}
& \text { 1) } t_{0} \succsim t \succsim t_{1} \Longrightarrow x\left(t, t_{0},\left(x_{0}, y_{0}\right)\right) \approx 0 \\
& \text { 2) } t_{1} \succsim t \Longrightarrow x\left(t, t_{0},\left(x_{0}, y_{0}\right)\right) \gtrsim 0
\end{aligned}
$$

Actually point 3 is true for $t$ not too large since we have a limit cycle.

Proof. Since the slow curve $\Pi$ is a trivial (or structural) "canard" ${ }^{3}$ of (3) the proof is very easy. We use the change of variable introduced by E. Benoît ([5]) in the case of non trivial "canards". Let us put :

$$
\xi=\varepsilon \ln (x)
$$

in the system (3). The new system, in the variables $(\xi, y)$, reads :

$$
\left\{\begin{array}{l}
\frac{d \xi}{d t}=2\left(1-\exp \left(\frac{\xi}{\varepsilon}\right)\right)-\frac{y}{0.1+\exp \left(\frac{\xi}{\varepsilon}\right)} \\
\frac{d y}{d t}=\left(\frac{\exp \left(\frac{\xi}{\varepsilon}\right)}{0.1+\exp \left(\frac{\xi}{\varepsilon}\right)}-m\right) y
\end{array}\right.
$$

3. Recall that a "canard" in a slow-fast system is a solution which is for an appreciable duration in the halo of the attracting part of the slow curve and followed by an appreciable duration in the halo of the repelling part. 
As soon as $\xi$ is negative and non infinitesimal the quantity $x=\exp \left(\frac{\xi}{\varepsilon}\right)$ is infinitesimal and the system (4) is approximated by the system :

$$
\left\{\begin{array}{l}
\frac{d \widehat{\xi}}{d t}=2-10 \widehat{y} \\
\frac{d \widehat{y}}{d t}=-m \widehat{y}
\end{array}\right.
$$

that we can integrate explicitly from the initial condition $\left(0, \widehat{y_{0}}=y_{0}\right)$. One gets :

$$
\begin{gathered}
\widehat{y}\left(t, t_{0},\left(0, y_{0}\right)\right)=y_{0} \exp \left(-m\left(t-t_{0}\right)\right) \\
\widehat{\xi}\left(t, t_{0},\left(0, y_{0}\right)\right)=\int_{t_{0}}^{t}\left(2-10 y_{0} \exp \left(-m\left(s-t_{0}\right)\right)\right) d s
\end{gathered}
$$

That last quantity is decreasing as far as $\left(2-10 y_{0} \exp \left(-m\left(s-t_{0}\right)\right)\right)$ is negative, thus attains its minimum for :

$$
t^{*}=t_{0}+\frac{1}{m} \ln \left(5 y_{0}\right)
$$

and then increases up to the value 0 which is attained at time $t_{1}$ defined by :

$$
\int_{t_{0}}^{t_{1}}\left(2-10 y_{0} \exp \left(-m\left(s-t_{0}\right)\right)\right) d s=0
$$

It is now just a routine verification to see that if

$$
\left(x\left(t, t_{0},\left(x_{0}, y_{0}\right)\right), y\left(t, t_{0},\left(x_{0}, y_{0}\right)\right)\right)
$$

is the solution of (3) with initial condition $\left(x_{0}, y_{0}\right)$ (such that $\xi_{0} \approx 0$ ) at time $t_{0}$ we have for $t_{0} \succsim t \lesssim t_{1}$ :

$$
\left(\varepsilon \ln \left(x\left(t, t_{0},\left(x_{0}, y_{0}\right)\right)\right), y\left(t, t_{0},\left(x_{0}, y_{0}\right)\right)\right) \approx\left(\widehat{\xi}\left(t, t_{0},\left(0, y_{0}\right)\right), \widehat{y}\left(t, t_{0},\left(0, y_{0}\right)\right)\right)
$$

which achieves the proof of the proposition.

Définition 2.2 The standard function $\varphi$ defined for standard $y_{0}$ by:

$$
\varphi\left(y_{0}\right)=\widehat{y}\left(t_{1},\left(0, y_{0}\right)\right)
$$

where $t_{1}$ is defined by (7) is called the entrance-exit function.

The entrance-exit function tells us, up to an infinitesimal, where a solution which entered in the halo of $\Pi^{+}$close to $\left(0, y_{0}\right)$ will leave the halo of $\Pi^{-}$. It can be computed with a very high accuracy using formulas (6) and (7). We compare in Tab. 1 the actual value of 


\begin{tabular}{|l|ll|ll|ll|ll|}
\hline$y_{0}$ & \multicolumn{2}{|c|}{0.4} & \multicolumn{2}{|c|}{0.5} & \multicolumn{2}{c|}{0.6} & \multicolumn{2}{|c|}{0.7} \\
\hline \hline$\varphi\left(y_{0}\right)$ & 8.12 & $10^{-2}$ & 5.35 & $10^{-2}$ & 3.57 & $10^{-2}$ & 2.38 & $10^{-2}$ \\
\hline$y_{\text {out }}$ & 8.33 & $10^{-2}$ & 5.45 & $10^{-2}$ & 3.57 & $10^{-2}$ & 2.37 & $10^{-2}$ \\
\hline
\end{tabular}

Table 1. Values of the parameters : $\varepsilon=0.05 ; m=0.7 ; x_{0}=0.05 ; \xi_{0}=0$

$y\left(t_{1},\left(x_{0}, y_{0}\right)\right)$ obtained by simulation and denoted by $y_{\text {out }}$ to the predicted value $\varphi\left(y_{0}\right)$ of the approximation. The approximation is quite good and sufficient for our following purposes.

Since this approximation is based on an approximation of the system (4) there is no reason that when we go back from the $\xi$ variable to the $x$ one through $x=\exp \left(\frac{\widehat{\xi}}{\varepsilon}\right)$ we obtain a good approximation of $x$. But we can be quite confident in a numerical simulation of (4) since in our case the values of $\xi$ range between -10 and 0 . Let us denote by $\xi_{\text {min }}$ the minimum of $\xi\left(t,\left(x_{0}, y_{0}\right)\right)$ obtained by a simulation of (4) (with an Euler-scheme with $\left.d t=10^{-4}\right)$ and compare $\exp \left(\frac{\xi_{\min }}{\varepsilon}\right)$ to the minimum, denoted by $x_{m i n}$, obtained by simulation of the system (2). This is done on Tab. 2. This good result in simulating

\begin{tabular}{|l|ll|ll|ll|ll|}
\hline$y_{0}$ & \multicolumn{2}{|c|}{0.4} & \multicolumn{2}{c|}{0.5} & \multicolumn{2}{c|}{0.6} & \multicolumn{2}{c|}{0.7} \\
\hline \hline $\exp \left(\frac{\xi_{\min }}{\varepsilon}\right)$ & 1.66 & $10^{-9}$ & 1.79 & $10^{-16}$ & 2.14 & $10^{-24}$ & 5.34 & $10^{-33}$ \\
\hline$x_{\min }$ & 1.62 & $10^{-9}$ & 1.69 & $10^{-16}$ & 1.90 & $10^{-24}$ & 4.31 & $10^{-33}$ \\
\hline
\end{tabular}

Table 2. Values of the parameters : $\varepsilon=0.05 ; m=0.7 ; x_{0}=0.05 ; \xi_{0}=\varepsilon \log \left(x_{0}\right)$

directly (2) is explained by the fact that around 0 the floating-point implementation of the real numbers on a computer is very precise. This point was considered in [14].

\subsection{The RMA model with immigration of the resource}

One way to prevent the resource $x$ of being too small in the RMA model is to introduce some immigration term : in the following model (8) we add the constant immigration rate $I$.

$$
\left\{\begin{aligned}
\frac{d x}{d t} & =\frac{1}{\varepsilon}\left(2 x(1-x)-\frac{x}{0.1+x} y\right)+I \\
\frac{d y}{d t} & =\left(\frac{x}{0.1+x}-m\right) y
\end{aligned}\right.
$$

Notice that we have put the term $I$ outside of the bracket. On Fig. 5 one sees the isoclines of (8) in the case $I=1$. The x-isocline

$$
\left\{(x, y): y=[2 x(1-x)+\varepsilon I] \frac{(0.1+x)}{x}\right\}
$$




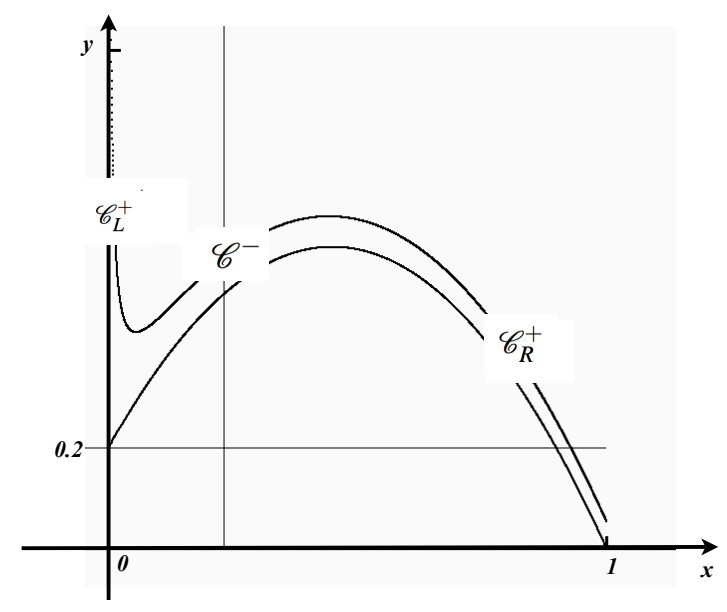

Figure 5. Slow curve : $I=1$.

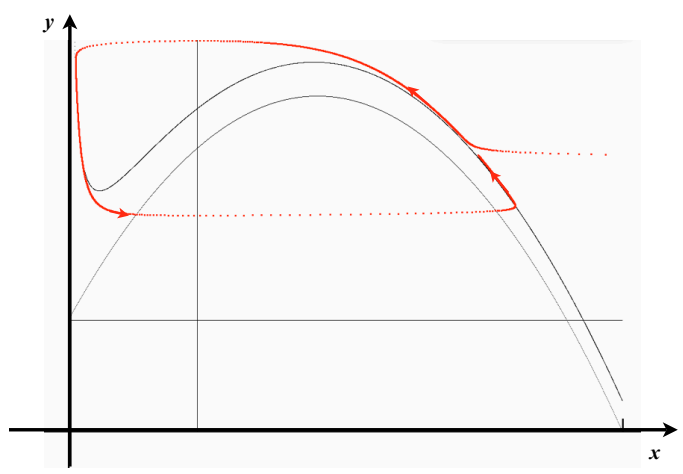

Figure 6. Limit cycle : $\varepsilon=0.05 ; I=1$.

is a cubic shaped curve which, when considered as the slow curve, is divided in three: $\mathcal{C}_{L}^{+}$ and $\mathcal{C}_{R}^{+}$for the attracting part, $\mathcal{C}^{-}$for the repelling part. When $I$ is limited, the term $\varepsilon I$ is infinitesimal and the x-isocline is in the halo of the parabola and the y-axis and $\mathcal{C}_{L}^{+}$is at a distance of the $\mathrm{x}$-axis of the order of $\varepsilon I$ except at the point $(0,0.2)$. The y-isocline is unchanged. There is still a limit cycle (see Fig. 6) and Fig. 7 shows its evolution with decreasing values of $I$. As in the case with no immigration the change of variable $\xi=\varepsilon \log (x)$ helps to understand the phase portrait. On Fig. 8 we have first simulated the model with $I=0$. This gives the blue stippled line in the $(\xi, y)$ variables going very far in the negative values; after that we simulated with the small value $I=2 \times 10^{-5}$; 

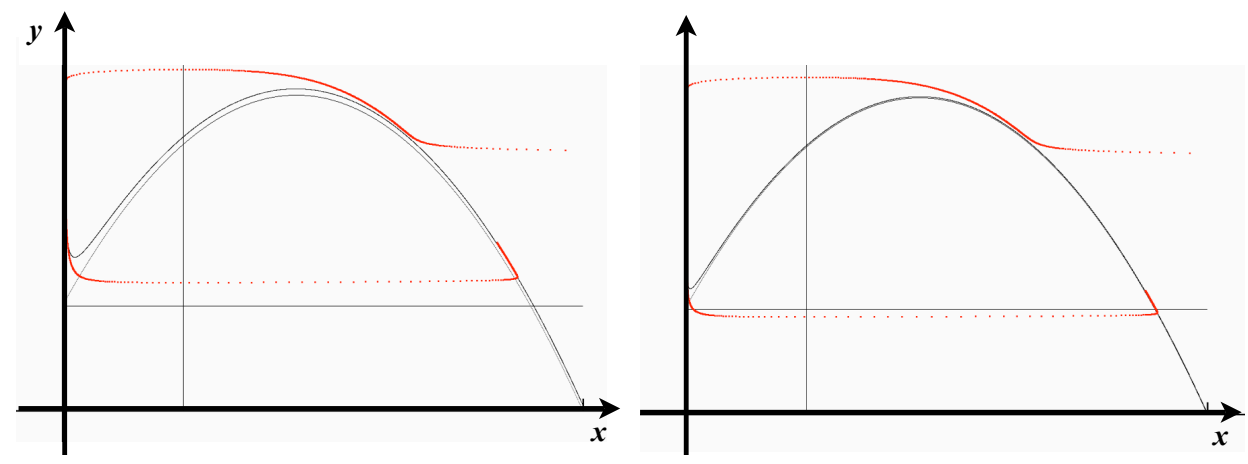

Figure 7. Limit cycle : $\varepsilon=0.05 ; I=0.2$ and $I=0.04$.

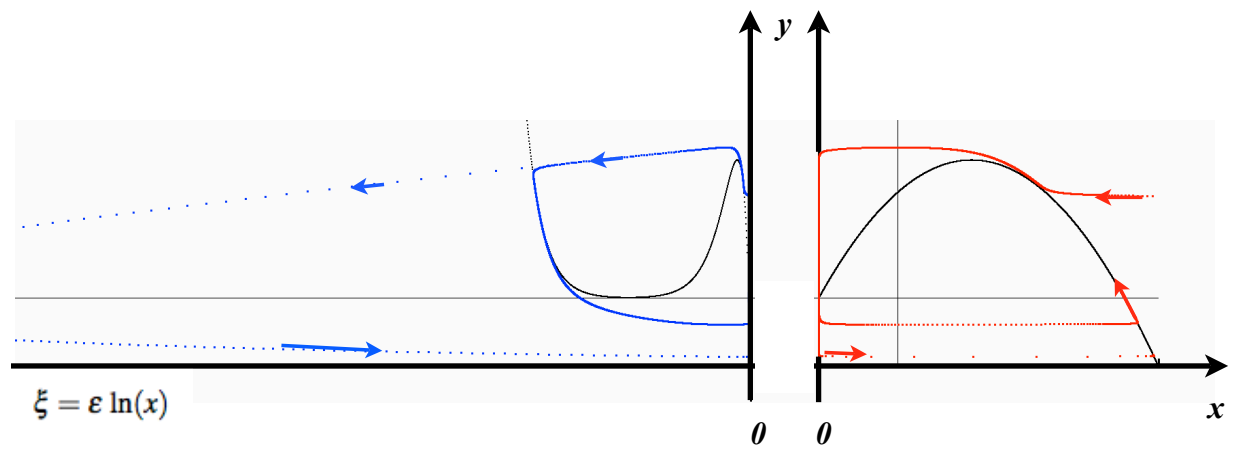

Figure 8. Immigration with a very small immigration rate $: \varepsilon=0.05 ; I=0.00002$.

in the $(x, y)$ variable we see a significant difference in the value of the minimum of $y(t)$ which is explained in the $(\xi, y)$ variables by the shape of the isocline. We see (an can prove easily) that when $I$ is different from 0 the minimum of $x(t)$ is of the order of $\varepsilon I$. Regarding the "atto-fox problem" this is very different from the exponentially small value with respect to $\varepsilon$ obtained when $I=0$. 


\section{The Rosenzweig-MacArthur model on two patches}

We consider now two identical patches with two identical populations. In absence of migration the model is :

$$
\left\{\begin{aligned}
\frac{d x_{1}}{d t} & =\frac{1}{\varepsilon}\left(x_{1} g\left(x_{1}\right)-h\left(x_{1}\right) y_{1}\right) \\
\frac{d y_{1}}{d t} & =\left(h\left(x_{1}\right)-m\right) y_{1} \\
\frac{d x_{2}}{d t} & =\frac{1}{\varepsilon}\left(x_{2} g\left(x_{2}\right)-h\left(x_{2}\right) y_{2}\right) \\
\frac{d y_{2}}{d t} & =\left(h\left(x_{2}\right)-m\right) y_{2}
\end{aligned}\right.
$$

This is a trivial model with two non interacting populations. On each patch we have an "atto-fox problem". But we can imagine that each patch receives from the other one, through migration, a certain number of individuals which prevent it from too low densities. How do we modelize this migration ? Consider the functions :

$$
\begin{aligned}
(x, y) & \mapsto \nu_{x}(x, y) \\
(x, y) & \mapsto \nu_{y}(x, y)
\end{aligned}
$$

which represent the rate of emigration from one patch to the other one of the resource or the consumer. If we assume that all the resource or consumer emigrating from one patch goes to the other patch we have the model :

$$
\left\{\begin{aligned}
\frac{d x_{1}}{d t} & =\frac{1}{\varepsilon}\left(x_{1} g\left(x_{1}\right)-h\left(x_{1}\right) y_{1}\right)+\nu_{x}\left(x_{2}, y_{2}\right)-\nu_{x}\left(x_{1}, y_{1}\right) \\
\frac{d y_{1}}{d t} & =\left(h\left(x_{1}\right)-m\right) y_{1}+\nu_{y}\left(x_{2}, y_{2}\right)-\nu_{y}\left(x_{1}, y_{1}\right) \\
\frac{d x_{2}}{d t} & =\frac{1}{\varepsilon}\left(x_{2} g\left(x_{2}\right)-h\left(x_{2}\right) y_{2}\right)+\nu_{x}\left(x_{1}, y_{1}\right)-\nu_{x}\left(x_{2}, y_{2}\right) \\
\frac{d y_{2}}{d t} & =\left(h\left(x_{2}\right)-m\right) y_{2}+\nu_{y}\left(x_{1}, y_{1}\right)-\nu_{y}\left(x_{2}, y_{2}\right)
\end{aligned}\right.
$$

Notice that, as wee did in the previous section, we choose to put the migration terms outside of the bracket following the term $\frac{1}{\varepsilon}$. In the sequel we shall call :

- One-patch system the system (3)

- Two-patches system the system (10)

The most widely considered model for migration is the linear one where the functions $\nu_{x}$ and $\nu_{y}$ are simply :

$$
\nu_{x}(x, y)=k_{x} x
$$




$$
\nu_{y}(x, y)=k_{y} y
$$

where $k_{x}$ and $k_{y}$ are some constants. This kind of model with linear migration (one also says linear dispersal or linear diffusion) has been considered both on the mathematical and ecological point of view mainly in the case of competition models where significant results where obtained (see [12] for a pioneering work in this direction). Not only the case of two patches was considered but also many patches and a continuum of patches, this last case being treated as a reaction-diffusion equation. One reason of this success is that cooperative systems are monotone which prevents the existence of limit cycles. In [13] the case of consumer-resource relationship on two patches with linear migration of the consumer is considered ; a lot of of interesting behaviors is observed but not in connection to our " atto-fox problem".

But, as we said, linear diffusion is just an approximation of what a migration model should be. The only certain fact regarding the functions $\nu_{x}(x, y)$ and $\nu_{y}(x, y)$ is that they must be non negative and that $\nu_{x}(0, y)=\nu_{y}(x, 0)=0$ : no individuals no emigration out of the patch is possible. Otherwise we can consider many different possibilities depending on the considered populations. For instance :

1) Under some threshold the population does not migrate, above it migrates at a constant rate which we modelize as $\nu_{x}(x, y)=0$ if $x \leq a$ and $\nu_{x}(x, y)=I$ otherwise $^{4}$.

2) When the population of resources is to low (under some threshold) the population of consumers decides to migrate.

3) For low populations the migration of the consumers is linear with some saturation for large values.

4) etc.

In section 3.3 we will consider the case 1 of the above enumeration which has some legitimacy regarding modelling and will show that it is to some extend mathematically tractable.

\subsection{Linear migration and homogenization.}

As we said previously, if when the density of the resource is very low on one of the patches and is high enough on the other one to produce emigration the atto-fox problem could be avoided. But this will work only if the two patches are not synchronized in approximately the same state and, moreover, the difference of phases between the two patches is stable. This is a problem since we have the intuition that migration between

4. In the world of micro-organisms there is a mechanism called "quorum sensing" by which certain species of micro-organisms change their behavior when a certain threshold is attained. 


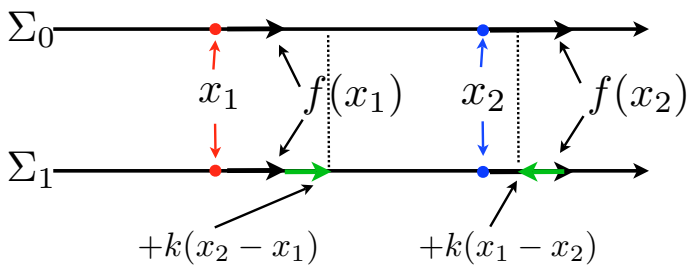

Figure 9. Linear migration in dim. 1

two patches could produce some kind of homogenization. Actually this is true for linear diffusion in dimension one. Consider the two linearly coupled one dimension systems :

$$
\Sigma_{k} \quad\left\{\begin{array}{l}
\frac{d x_{1}}{d t}=f\left(x_{1}\right)+k x_{2}-k x_{1} \\
\frac{d x_{2}}{d t}=f\left(x_{2}\right)+k x_{1}-k x_{2}
\end{array}\right.
$$

For $k=0$ the systems are uncoupled. Let $x\left(t, x_{1}(0), k=0\right)$ and $x\left(t, x_{2}(0), k=0\right)$ with $x_{1}(0)<x_{2}(0)$ be two solutions of $\Sigma_{0}$ and consider $x\left(t, x_{1}(0), k\right)$ and $x\left(t, x_{2}(0), k\right)$ with $k>0$ the solutions of $\Sigma_{k}$ with the same initial conditions. Fig. 9 shows why one has for $t>0$ :

$$
\begin{aligned}
& x\left(t, x_{1}(0), 0\right)<x\left(t, x_{1}(0), k\right) \\
& x\left(t, x_{2}(0), k\right)<x\left(t, x_{2}(0), 0\right)
\end{aligned}
$$

We see that thanks to the diffusion the speed of $x\left(t, x_{1}(0), k\right)$ is greater than the speed of $x\left(t, x_{1}(0), 0\right)$ and conversely for $x\left(t, x_{2}(0), k\right)$ and $x\left(t, x_{2}(0), k\right)$. This fact admits a generalization to higher dimensions, the convex interval

$$
\left[x\left(t, x_{1}(0), 0\right), x\left(t, x_{2}(0), 0\right)\right]
$$

which is clearly invariant by the flow, being replaced by an invariant familly of convex sets. On Fig. 10 we have drawn the flow lines of some flow $\Phi$ generated by the differential system in the plane :

$$
\left\{\begin{array}{l}
\frac{d x}{d t}=f(x, y) \\
\frac{d y}{d t}=g(x, y)
\end{array}\right.
$$


System (13) represents two such systems coupled by linear diffusion :

$$
\left\{\begin{aligned}
\frac{d x_{1}}{d t} & =f\left(x_{1}, y_{1}\right)+k\left(x_{2}-x_{1}\right) \\
\frac{d y_{1}}{d t} & =g\left(x_{1}, y_{1}\right)+k\left(y_{2}-y_{1}\right) \\
\frac{d x_{2}}{d t} & =f\left(x_{2}, y_{2}\right)+k\left(x_{1}-x_{2}\right) \\
\frac{d y_{2}}{d t} & =g\left(x_{2}, y_{2}\right)+k\left(y_{1}-y_{2}\right)
\end{aligned}\right.
$$

A solution of system (13) can be visualised as two corks floating on the flow and con-

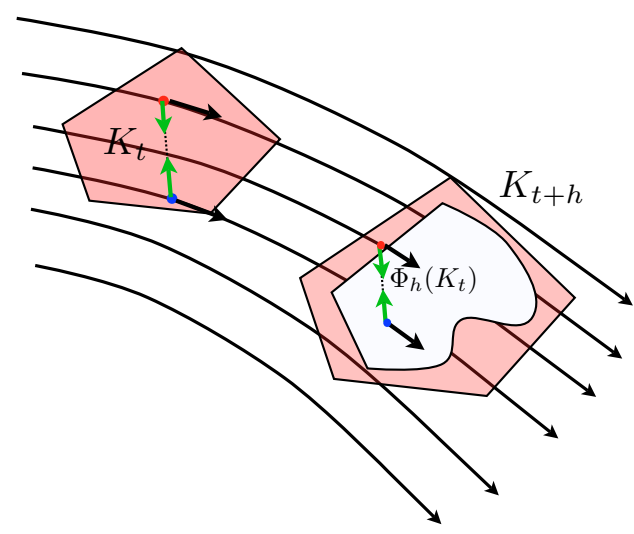

Figure 10. Two corks on a flow.

nected by a rubber which creates a relative velocity (green arrows on Fig. 10) directed along the line joining the two corks and of intensity proportional to their distance. From this remark we see that if $K$ is a convex set invariant by the flow, i. e. such that the vector field points inward, two corks connected by a rubber which are initially inside $K$ will remain in $K$ since the velocity of a cork which is on the boundary of $K$, being the sum of two velocities directed inward is directed inward. This works also when $t \mapsto K_{t}$ is a family of convex sets invariant by the flow (i.e. for $h>0$ one has $\Phi_{h}\left(K_{t}\right) \subset K_{t+h}$ ) and if the initial condition is such that $\left(x_{1}(0), y_{1}(0)\right) \in K_{0}$ and $\left(x_{2}(0), y_{2}(0)\right) \in K_{0}$ then for every positive $t$ we have $\left(x_{1}(t), y_{1}(t)\right) \in K_{t}$ and $\left(x_{2}(t), y_{2}(t)\right) \in K_{t}$; in other words the corks are trapped in the family of convex. This generalizes to an arbitrary number of patches or a continuum (see [21]). From this we see that if the differential system (12) 
has an equilibrium G.A.S. which admits a Liapunov function with convex level lines then on the two patches the solutions will converge to this equilibrium and the homogenization will be perfect. But, otherwise, homogenization is not the rule. This is the case when the system admits a limit cycle.

\subsection{Linear migration in the RMA model}

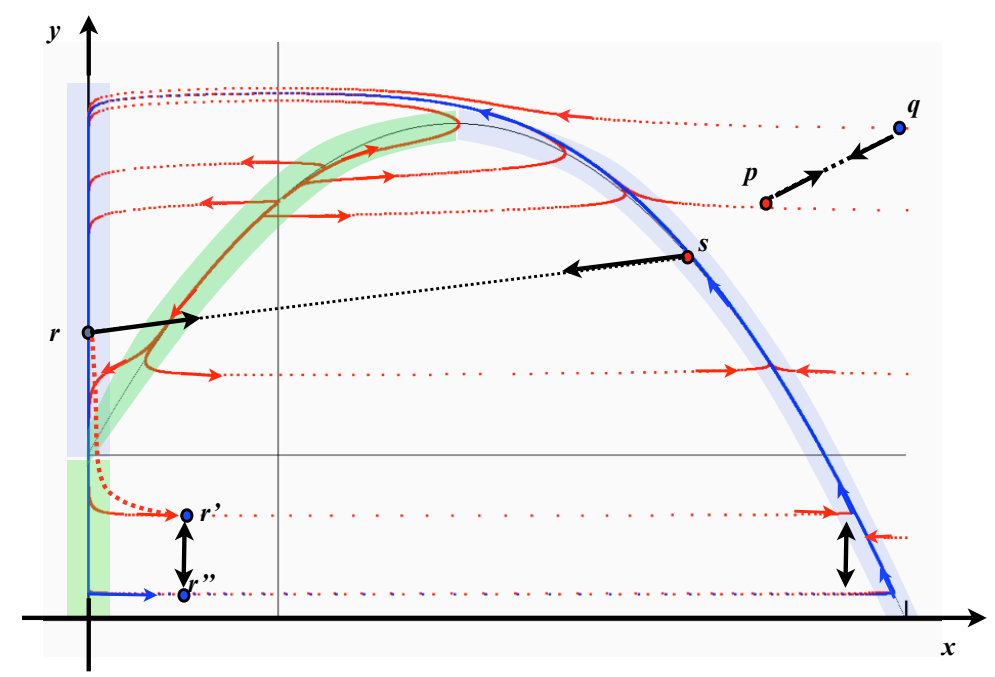

Figure 11. Two pairs $p, q$ and $r, s$ of corks;

Consider the RMA model, in the case of a limit cycle, with linear migration.

$$
\left\{\begin{aligned}
\frac{d x_{1}}{d t} & =\frac{1}{\varepsilon}\left(x_{1} g\left(x_{1}\right)-h\left(x_{1}\right) y_{1}\right)+k\left(x_{2}-x_{1}\right) \\
\frac{d y_{1}}{d t} & =\left(h\left(x_{1}\right)-m\right) y_{1}+k\left(y_{2}-y_{1}\right) \\
\frac{d x_{2}}{d t} & =\frac{1}{\varepsilon}\left(x_{2} g\left(x_{2}\right)-h\left(x_{2}\right) y_{2}\right)+k\left(x_{1}-x_{2}\right) \\
\frac{d y_{2}}{d t} & =\left(h\left(x_{2}\right)-m\right) y_{2}+k\left(y_{1}-y_{2}\right)
\end{aligned}\right.
$$

This is a model of two linearly coupled oscillators. It is known that the analysis of the dynamic properties of two coupled oscillators might be difficult and a very large literature is devoted to this subject. Since the RMA model is in the plane the cork metaphor works. If we assume that $k$ is limited the relative motion of the corks with respect to the flow is 


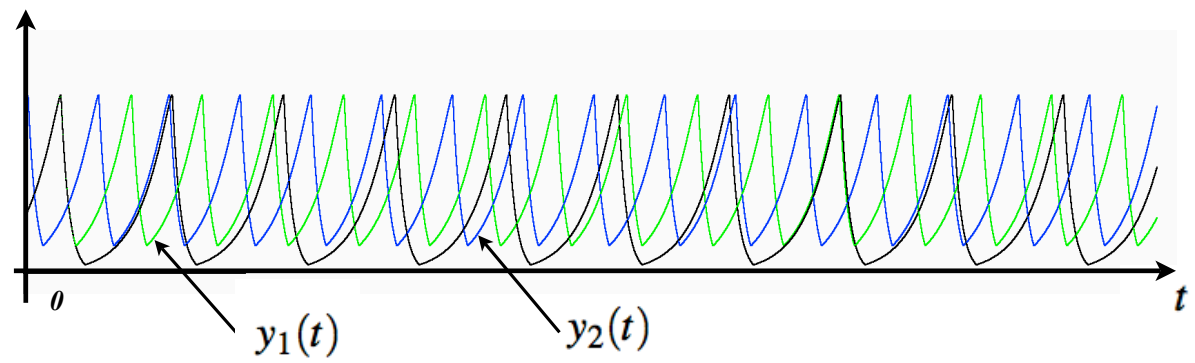

Figure 12. $y(t)$ in black, $y_{1}(t)$ in green, $y_{2}(t)$ in blue. Parameters of 3.2.1.

just limited and does not affect the fast motion of the corks: see the pair $(p, q)$ on Fig. 11. If we consider now the pair $(r, s)$ the motion of $r$ and $s$ will remain in the halo of the slow curve since $r$ and $s$ are in the stable part. But the distance of $r$ to the $y$ axis will not be exponentially small as in the absence of rubber but of the order of magnitude of $\varepsilon k$. As a consequence of this, the exit of $r$ will occur much faster at a point $r^{\prime}$ much higher than the point $r^{\prime \prime}$ of exit in absence of migration. This difference $\left\|r^{\prime} r^{\prime \prime}\right\|$ will be responsible of a reduction of the duration needed to move up to the summit of the slow curve and, by the way, of a reduction of the period. In other words even a small (but not exponentially small) migration will have an appreciable effect on the minimum of $y$ and on the period. This description is correct provided during the time needed by $r$ to move down the $y$ axis the point $s$ remains on the left part of the slow curve, let us say as far as $r$ and $s$ are in "phase oposition". This explains to some extend what we observe in the following simulations.

\subsubsection{Simulation : Linear migration 1}

The conventions are the following :

- In black $t \mapsto x(t)$ and $t \mapsto y(t)$ are solutions of the one-patch system (3).

- In green $t \mapsto x_{1}(t)$ and $t \mapsto y_{1}(t)$ are solutions on the first patch of the two-patches system (10).

- In blue $t \mapsto x_{2}(t)$ and $t \mapsto y_{2}(t)$ are solutions on the second patch of the $t w o$ patches system (10).

\section{Parameters.}

- Growth parameters :

$\varepsilon=0.05 ; m=0.7$

- Migration parameter (small linear migration): $k=10^{-6}$

- Initial conditions :

$x(0)=0.9 ; y(0)=0.2 \quad x_{1}(0)=0.9 ; y_{1}(0)=0.2 \quad x_{2}(0)=0.5 ; y_{2}(0)=0.6$ 


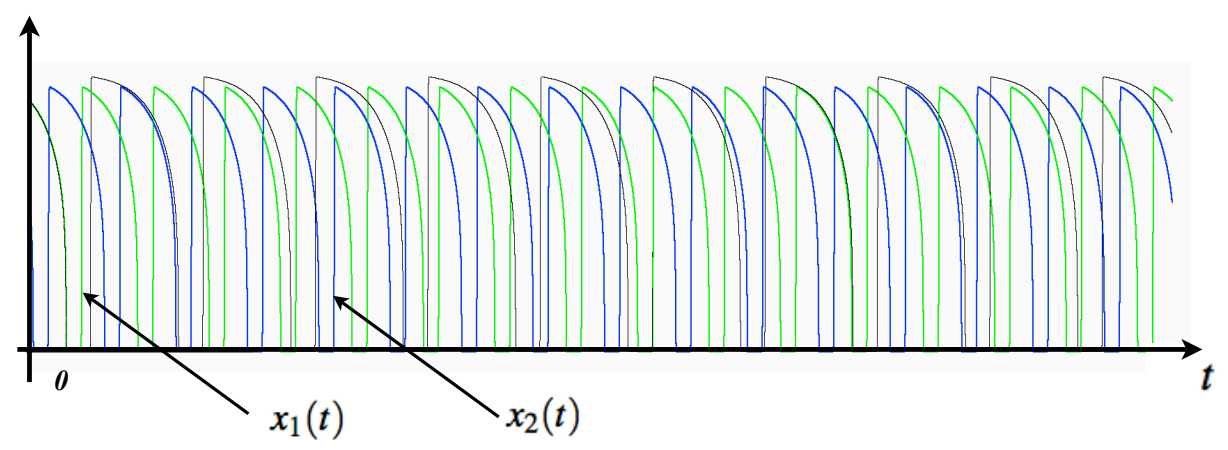

Figure 13. $x(t)$ in black, $x_{1}(t)$ in green, $x_{2}(t)$ in blue. Parameters of 3.2.1.

- Duration of the simulation : 200 units of time.

The results of the simulation are shown on Fig. 12 and 13 that we comment now. We begin with the simulations regarding $y$ which are easier to understand.

On Fig. 12 the solution $t \mapsto y(t)$ of the one-patch system oscillates with a period of approximately 20 units of time and takes a minimal value close to 0 . The solution $t \mapsto y_{1}(t)$ of the two-patches system (10) is identical to $y(t)$ for approximately 10 units of time ; suddenly it becomes different (the green curve departs from the black one) and oscillates now approximately two times faster than on the one-patch system (3); the new minimum for $y_{1}(t)$ is significantly bigger than 0 ; the solution, $t \mapsto y_{2}(t)$ on the other patch of the two-patches system (10) has a phase opposed to that of $t \mapsto y_{1}(t)$. On Fig. 13 we see $x(t)$ (in black), $x_{1}(t)$ and $x_{2}(t)$ in green and blue. We can make the same comments than for $y$ about periodicity of $x_{i}(t)$. Moreover one sees that $x(t)$ and $x_{i}(t)$ are apparently equal to 0 during a part of the cycle but the values of the minima are quite different for $x$ and $x_{i}(t)$ :

$-\min x(t)$ is of the order of $10^{-26}$

$-\min x_{1}(t)=\min x_{2}(t)$ is of the order of $10^{-8}$

They are not of the same order of magnitude which is important regarding the biological interpretation. In the absence of migration we have certainly an "atto-fox" problem, with migration it depends on the number of individuals represented by one unit of $x$.

Unfortunately we do not know if this simulation is a simulation of a stable limit cycle. In other word we have no guarantee that what we observe is not just a (long) transient.

\subsubsection{Simulation : Linear migration 2}

Since it is not realistic to assume that the coefficients of the linear migration are the same for the consumer and the resource we have considered a new simulation. This new simulation is performed with exactly the same parameters than the simulation 3.2.1 except that the parameters of the linear migration terms are different for $x$ and $y$. Namely 


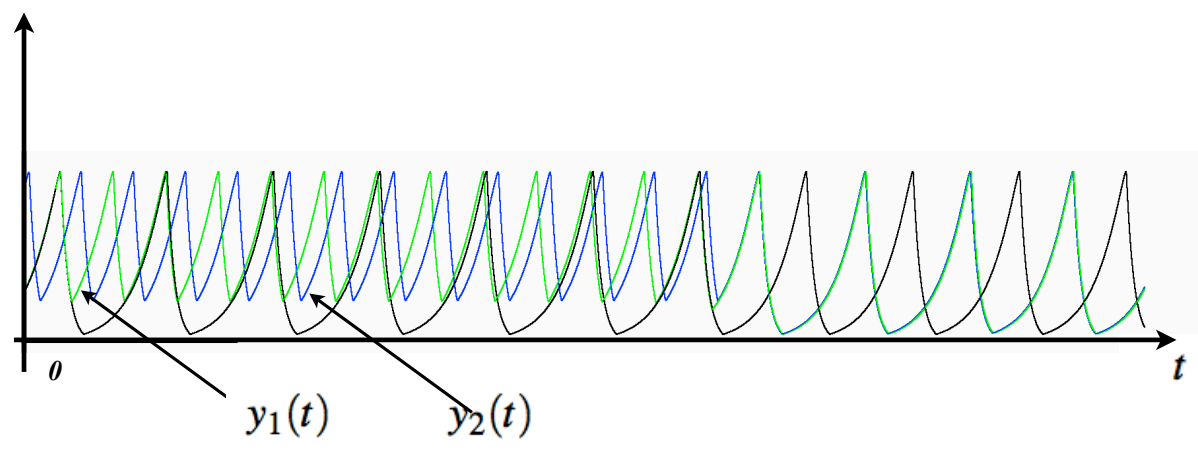

Figure 14. $y(t)$ in black, $y_{1}(t)$ in green, $y_{2}(t)$ in blue. Parameters of 3.2.2.

$k_{x}=0.01$ and $k_{y}=0.0005$. The migration for $x$ is much stronger than for $y$ and the interpretation in terms of corks is no longer valid.

The result is shown on Fig. 14. We see that $y_{1}(t)$ and $y_{2}(t)$ which have opposite phases at the beginning progressively synchronize ; when they are synchronized (we only see $y_{1}(t)$ in green, the blue $y_{2}(t)$ being plotted the first is hidden) the motion is exactly the same than the motion on the one-patch system (3) .

\subsection{Nonlinear migration of the resource}

We examine in this section a mathematically simpler situation than the linear migration one which, fortunately, has some biological significance. We suppose that there is only migration of the resource and that this migration is non linear and defined by a step function :

$$
\text { If } x>a \text { then } \nu_{x}=I \text { else } \nu_{x}=0
$$

This means that once the resource is above some threshold $a$ there is a migration which rate is independent of $x$. This last point is probably non realistic but acceptable as a first approximation. Moreover we shall assume that the immigration term $I$ is not too big (i.e limited). We have seen in section 2.3 that even if $I$ is small (but not exponentially small), regarding modelling purposes, it could make a big difference with no immigration at all : the minimum of $x(t)$ will be of the order of $\varepsilon I$, not exponentially small. 


\subsubsection{Two patches with "spontaneous migration"}

We call "spontaneous migration model" (with reference to spontaneous generation ) the model :

$$
\left\{\begin{aligned}
\frac{d \hat{x}_{1}}{d t} & =\frac{1}{\varepsilon}\left(\hat{x}_{1} g\left(\hat{x}_{1}\right)-\mu\left(\hat{x}_{1}\right) \hat{y}_{1}\right)+I \\
\frac{d \hat{y}_{1}}{d t} & =\left(\mu\left(\hat{x}_{1}\right)-m\right) \hat{y}_{1} \\
\frac{d \hat{x}_{2}}{d t} & =\frac{1}{\varepsilon}\left(\hat{x}_{2} g\left(\hat{x}_{2}\right)-\mu\left(\hat{x}_{2}\right) \hat{y}_{2}\right)+I \\
\frac{d \hat{y}_{2}}{d t} & =\left(\mu\left(\hat{x}_{2}\right)-m\right) \hat{y}_{2}
\end{aligned}\right.
$$

These are two uncoupled copies of the system (8) which was studied in section 2.3 with. We say "spontaneous migration" because the model does not say how are generated the resource migrants which are contained in the terms $I$. We do not know if such a system has some biological relevance; it is just defined for mathematical purposes. This system is trivial and we know that for suitable values of the parameters every solution (except for a set of initial conditions which is of codimention two) converges to a periodic solution. In the strictly positive orthant $\left(\mathbb{R}^{*+}\right)^{4}$ there are an infinite number of periodic solutions differing only by the phase along the limit cycle of (8). Precisely let $t \mapsto(x(t), y(t)) ; t \in$ $[0 ; T]$ be some parametrisation of the limit cycle of (8). All the periodic solutions of (15) can be described as :

$$
\left(\hat{x}_{1}(t), \hat{y}_{1}(t), \hat{x}_{2}(t), \hat{y}_{2}(t)\right)=(x(t), y(t), x(t+c), y(t+c)) ; c \in[0, T[
$$

Définition 3.1 A periodic solution of (15) is said in phase-opposition iff :

$$
\begin{aligned}
& \left(\hat{x}_{1}(t), \hat{y}_{1}(t)\right) \in \operatorname{hal}\left(\Pi^{+} \cup \Pi^{-}\right) \Longrightarrow\left(\hat{x}_{2}(t), \hat{y}_{2}(t)\right) \in \operatorname{hal}\left(\mathcal{C}_{R}^{+}\right) \\
& \left(\hat{x}_{2}(t), \hat{y}_{2}(t)\right) \in \operatorname{hal}\left(\Pi^{+} \cup \Pi^{-}\right) \Longrightarrow\left(\hat{x}_{1}(t), \hat{y}_{1}(t)\right) \in \operatorname{hal}\left(\mathcal{C}_{R}^{+}\right)
\end{aligned}
$$

During a period the shadow ${ }^{5}$ of the mapping $t \mapsto \hat{x}_{i}(t)(i=1,2)$ is the graph of a function which is continuous except at the time $t_{i}^{*}$ where $x$ jumps from $\mathcal{C}_{R}^{+}$to $\operatorname{hal}\left(\Pi^{+}\right)$ and $t_{i}^{* *}$ where $x$ jumps from hal $\left(\Pi^{-}\right)$to $\mathcal{C}_{R}^{+}$. These times are called jump-times.

It is easy to see that by adequate tuning of the parameter $m$ the model (15) has periodic solutions in phase-opposition.

5. Recall that the shadow of a set (in our case the graph of $t \mapsto x(t)$ ) is the standardization of its halo. 


\subsubsection{Two patches with threshold migration}

In this section we compare periodic solutions of the model (15) of the previous section with solutions of the model :

$$
\left\{\begin{aligned}
\frac{d x_{1}}{d t} & =\frac{1}{\varepsilon}\left(x_{1} g\left(x_{1}\right)-\mu\left(x_{1}\right) y_{1}\right)+\nu\left(x_{2}\right)-\nu\left(x_{1}\right) \\
\frac{d y_{1}}{d t} & =\left(\mu\left(x_{1}\right)-m\right) y_{1} \\
\frac{d x_{2}}{d t} & =\frac{1}{\varepsilon}\left(x_{2} g\left(x_{2}\right)-\mu\left(x_{2}\right) y_{2}\right)+\nu\left(x_{1}\right)-\nu\left(x_{2}\right) \\
\frac{d y_{2}}{d t} & =\left(\mu\left(x_{2}\right)-m\right) y_{2}
\end{aligned}\right.
$$

with :

$$
\begin{aligned}
& -\varepsilon \approx 0 \\
& -g(x)=2(1-x) \\
& -\mu(x)=\frac{x}{0.1+x} \\
& -m=0.7 \\
& -\nu(x)=I \text { if } x>a=0.2 \text { else } \nu=0 \text { with } I \text { limited. }
\end{aligned}
$$

Since there is no migration with $y$ we have simplified the notation $\nu_{x}$ to simply $\nu$. We prove that if an initial condition for system (16) is such that the corresponding solution of (15) is in phase-opposition then the solution of (16) will remain close to the solution of (15) during a long transient. Precisely :

Proposition 3.2 Let $\left(\hat{x_{1}}(t), \hat{y}_{1}(t), \hat{x}_{2}(t), \hat{y}_{2}(t)\right)$ be a periodic solution of $(15)$ in phaseopposition ; let $\left(x_{1}(t), y_{1}(t), x_{2}(t), y_{2}(t)\right)$ be a solution of (16) such that :

$$
\left(x_{1}(0), y_{1}(0), x_{2}(0), y_{2}(0)\right) \approx\left(\hat{x_{1}}(0), \hat{y}_{1}(0), \hat{x}_{2}(0), \hat{y}_{2}(0)\right)
$$

then for every limited $t$ :

$$
\left(x_{1}(t), y_{1}(t), x_{2}(t), y_{2}(t)\right) \approx\left(\hat{x_{1}}(t), \hat{y}_{1}(t), \hat{x}_{2}(t), \hat{y}_{2}(t)\right)
$$

except in the halo of jump-times. Moreover

$$
\hat{x}_{i}(t) \approx 0 \Longrightarrow \varepsilon \log \left(\hat{x}_{i}(t)\right) \approx \varepsilon \log \left(x_{i}(t)\right)
$$

Sketch of the proof Recall that $\mathcal{C}_{R}^{+}$is at an infinitesimal distance of the parabola. Assume that the initial condition is such that the point $\left(x_{1}(0), y_{1}(0)\right)$ and the point $\left(x_{2}(0), y_{2}(0)\right)$ both belong to $\mathbf{h a l}\left(\mathcal{C}_{R}^{+}\right)$. On Fig.15 the point $\left(x_{1}(0), y_{1}(0)\right)$ is represented by a red dot and the point $\left(x_{2}(0), y_{2}(0)\right)$ by a blue dot ; the point $\left(\hat{x}_{1}(0), \hat{y}_{1}(0)\right)$ is represented by a red star and the point $\left(\hat{x}_{2}(0), \hat{y}_{2}(0)\right)$ by a blue star. It will be clear later that these conditions are not restrictive. 


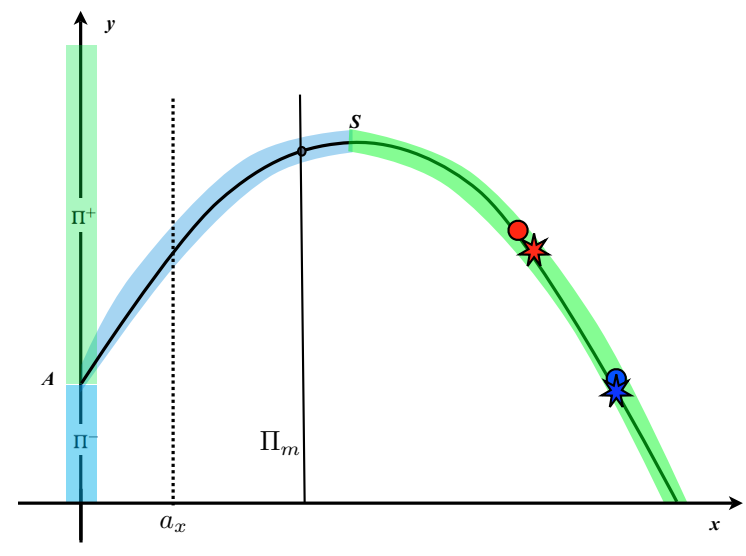

Figure 15. Initial condition. The stars represent the solution of the uncoupled system with spontaneous immigration. The dots represent the solution of the coupled system (16). Initial conditions are infinitesimally close together.

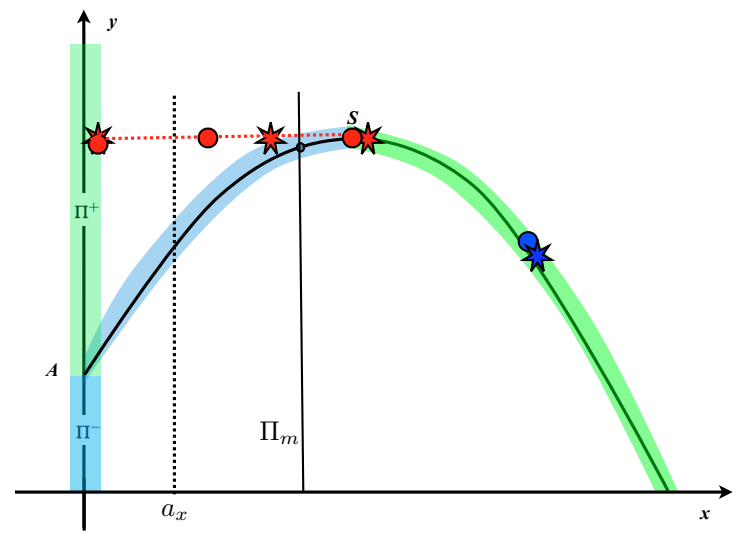

Figure 16. During the first jump. The two pairs : (red dot, red star) and (blue dot) (blue star) move up along $\mathcal{C}_{R}^{+}$until the "red pair" enters the halo of the summit of the parabola. The "red pair" jumps. During the jump the red dot and the red star are no longer equivalent but are again after the jump. 
1) Before the first “jump”(Fig.15). As long as $\left(x_{i}(t), y_{i}(t)\right)(i=1,2)$ are in $\operatorname{hal}\left(\mathcal{C}_{R}^{+}\right)$the equations for $\left(x_{i}, y_{i}\right)$ are :

$$
\left\{\begin{aligned}
\frac{d x_{i}}{d t} & =\frac{1}{\varepsilon}\left(x_{i} g\left(x_{i}\right)-\mu\left(x_{i}\right) y_{i}\right) \\
\frac{d y_{i}}{d t} & =\left(\mu\left(x_{i}\right)-m\right) y_{i} \quad i=1,2
\end{aligned}\right.
$$

since both are on the right of the migration threshold the two migration compensate. For $\left(\hat{x}_{i}, \hat{y}_{i}\right)$ the system is:

$$
\left\{\begin{aligned}
\frac{d \hat{x}_{i}}{d t} & =\frac{1}{\varepsilon}\left(\hat{x}_{i} g\left(\hat{x}_{i}\right)-\mu\left(\hat{x}_{i}\right) \hat{y}_{i}+\varepsilon I\right) \\
\frac{d \hat{y}_{i}}{d t} & =\left(\mu\left(\hat{x}_{i}\right)-m\right) \hat{y}_{i} \quad i=1,2
\end{aligned}\right.
$$

and the slow curve of (18) is close $^{6}$ to $\mathcal{P}^{+}$; since the equation for $y$ and $\hat{y}$ are the same the dots and the stars remain close until the first jump occurs.

2) First "jump"(Fig.16). The value of the first jump-time $t_{1}$ is the same for $\left(x_{1}, y_{1}\right)$ and $\left(\hat{x}_{1}, \hat{y}_{1}\right)$. During an infinitesimal duration the star and the dot might be separated but after they are again close.

3) After the "first jump" and before the "second jump"(Fig.17) Before the second "jump" of $\left(\hat{x}_{1}, \hat{y}_{1}\right)$ the point $\left(\hat{x}_{2}, \hat{y}_{2}\right)$ remains in $\mathbf{h a l}\left(\mathcal{C}_{R}^{+}\right)$and the equations are :

$$
\begin{aligned}
& \left\{\begin{aligned}
\frac{d x_{1}}{d t} & =\frac{1}{\varepsilon}\left(x_{1} g\left(x_{1}\right)-h\left(x_{1}\right) y_{1}+\varepsilon I\right) \\
\frac{d y_{1}}{d t} & =\left(h\left(x_{1}\right)-m\right) y_{1} \\
\frac{d x_{2}}{d t} & =\frac{1}{\varepsilon}\left(x_{2} g\left(x_{2}\right)-h\left(x_{2}\right) y_{2}-\varepsilon I\right) \\
\frac{d y_{2}}{d t} & =\left(h\left(x_{2}\right)-m\right) y_{2}
\end{aligned}\right. \\
& \left\{\begin{aligned}
\frac{d \hat{x}_{i}}{d t} & =\frac{1}{\varepsilon}\left(\hat{x}_{i} g\left(\hat{x}_{i}\right)-h\left(\hat{x}_{i}\right) \hat{y}_{i}+\varepsilon I\right) \\
\frac{d \hat{y}_{i}}{d t} & =\left(h\left(\hat{x}_{i}\right)-m\right) \hat{y}_{i} \quad i=1,2
\end{aligned}\right.
\end{aligned}
$$

The first two equations of (19) are exactly the same than those of (20). Thus $\left(x_{1}, y_{1}\right)$ and $\left(\hat{x}_{1}, \hat{y}_{1}\right)$ will remain close together also in the $\xi=\varepsilon \log (x)$ variables which means that the minimum of $x_{1}$ during this period is of the same order of magnitude than the minimum of $\hat{x}_{1}$.

6. We use close in place of infinitesimally close 


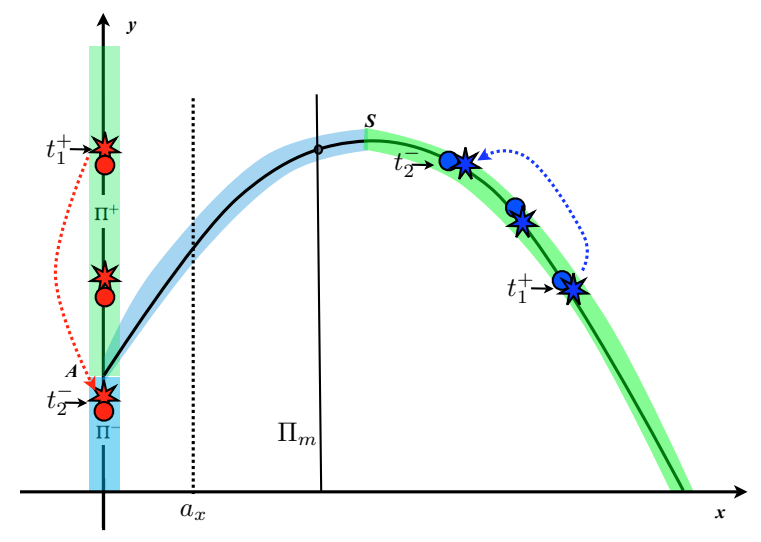

Figure 17. From $t_{1}^{+}$to $t_{2}^{-}$along $\Pi$. After the first jump of the "red pair" from $\mathcal{C}_{R}^{+}$to hal( $\left.\Pi\right)$ and before its jump from hal(П) to $\mathcal{C}_{R}^{+}$the "blue pair" moves into the halo of $\mathcal{C}_{R}^{+}$to some point which is not the summit of the parabola.

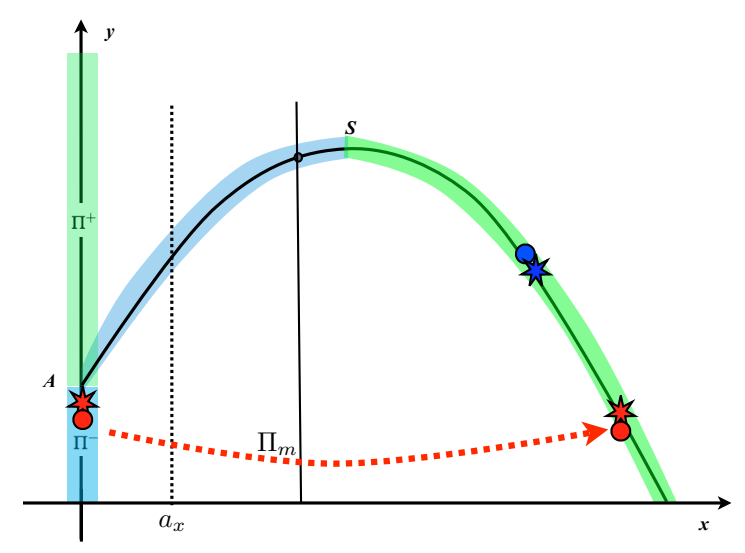

Figure 18. After the second jump of $x_{1}$. The situation is now the same than in Fig. 15 with the "red pair" exchange with the "blue pair". 


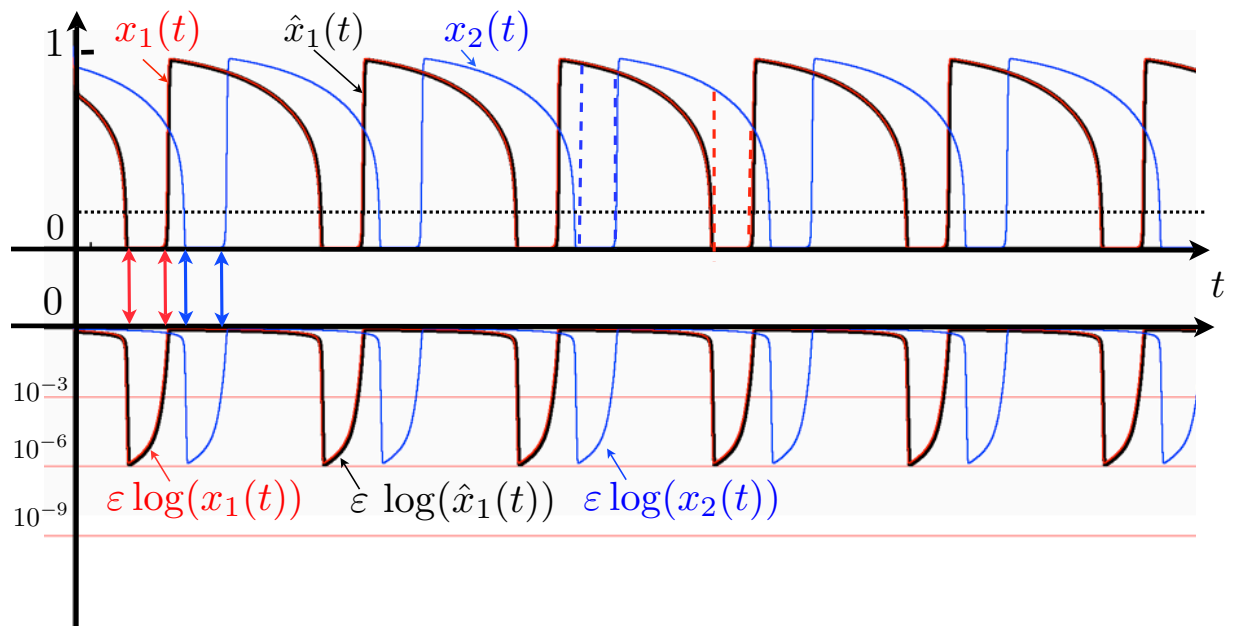

Figure 19. Models (15) and (16) $: \varepsilon=0.05 ; m=0.7 ; a=0.2 ; I=0.0001$. Initial conditions : $\left(x_{1}(0)=\hat{x}_{1}(0)=1 ; y_{1}(0)=\hat{y}_{1}(0)=0.4\right)$ and $\left(x_{2}(0)=\hat{x}_{2}(0)=1 ; y_{2}(0)=\right.$ $\left.\hat{y}_{2}(0)=0.2\right)$

4) The second "jump" and after (Fig.18) Let $t_{2}$ be the "time" of the second jump of $\hat{x}_{1}(t)$. Since the first two equations of (19) are those of (18) as long as $x_{1}<0.2$ both $\left(\hat{x}_{1}, \hat{y}_{1}\right)$ and $\left(x_{1}, y_{1}\right)$ will jump from hal $(\Pi)$ at the same time and will reach hal $\left(\mathcal{C}_{R}^{+}\right)$being close.

5) From the second "jump to the fourth one" We are now in the same position as at step one with 2 ("blue pair") in place of 1 ("red pair") ; thus after two more "jump" we recover the situation of step 1 .

6) Last step By external recurrence we can repeat steps 1 to 5 a limited number of time in order to attain any limited real $t$ and the proposition is proved.

\subsubsection{Simulations}

We comment here Fig. 19 to 23 which illustrate the previous result.

- Fig. 19. We represent the $x$ variables (above) and the $\varepsilon \log (x)$ variables (below) with respect to time. We have represented a simulation of $t \mapsto \hat{x}_{1}(t)$ of the model with spontaneous migration (15) as a very "thick black" curve. After that we have drawn $t \mapsto x_{1}(t)$ in "normal red" and $t \mapsto x_{2}(t)$ in "normal blue" of the model with nonlinear migration (16). We see that $x_{1}(t)$ is so close to $\hat{x}_{1}(t)$ that the "normal red" line is "contained" within the thickness of the "thick black" one. This can be seen on a computer if one enlarges the picture.

The parameters and initial conditions are :

- $m=0.7 ; a=0.2 ; I=0.0001$ 
- $\left(x_{1}(0)=\hat{x}_{1}(0)=1 ; y_{1}(0)=\hat{y}_{1}(0)=0.4\right)$

- $\left(x_{2}(0)=\hat{x}_{2}(0)=1 ; y_{2}(0)=\hat{y}_{2}(0)=0.2\right)$

When $x_{1}$ (in red) seems to be 0 the corresponding interval for $\varepsilon \log \left(x_{1}\right)$ (in red) shows the exact value ; the minimum for $x_{1}(t)$ is around $10^{-6}$ that we assume to be big enough to avoid an "atto-fox problem". We have the same correspondence for the $x_{2}$ variable (in blue).

The blue curve seems to be a shift of the red one.

The black dashed horizontal line represents the threshold $a=0.2$; the two blue dashed vertical lines show that when $x_{2}(t)$ is small then $x_{1}(t)$ is above the threshold and vice versa when $x_{1}(t)$ is small.

- Fig. 20. Now we only represent $t \mapsto x_{1}(t)$ (red) and $t \mapsto x_{2}(t)$ (blue) but for a longer duration. The parameters are the same than in Fig. 20 except the initial condition for $y_{1}$ which is now : $y_{1}(0)=0.5$.

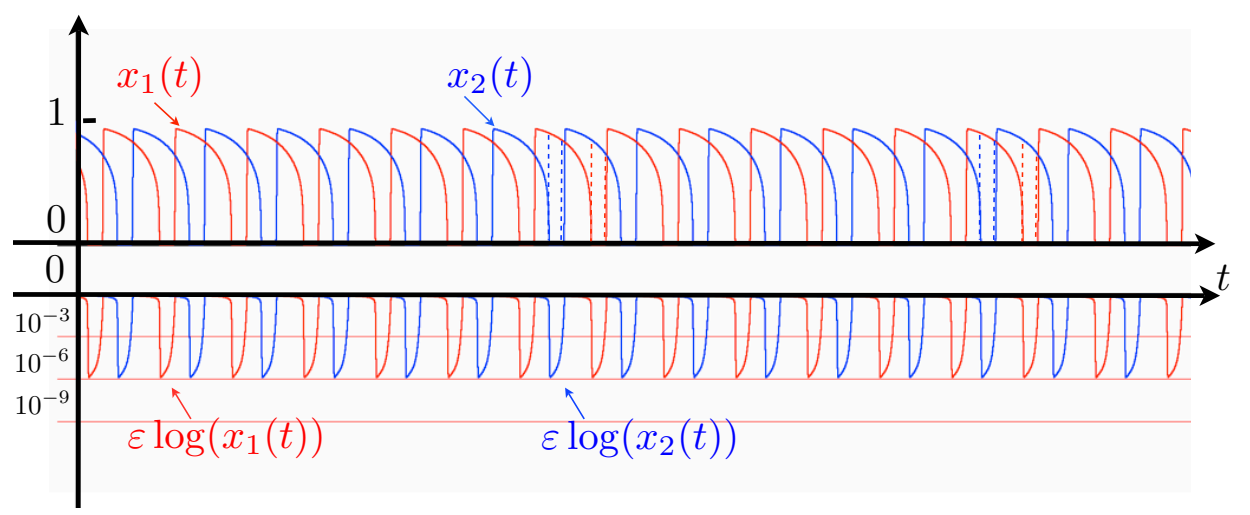

Figure 20. Model (16) : $\varepsilon=0.05 ; m=0.7 ; a=0.2 ; I=0.0001$. Initial conditions : $\left(x_{1}(0)=1 ; y_{1}(0)=0.5\right)$ and $\left(x_{2}(0)=1 ; y_{2}(0)=0.2\right)$

The picture shows that the shift between the two components $x_{1}(t)$ and $x_{2}(t)$ seems to be constant or, in other words the difference of phases seems to be constant.

For this initial conditions the intervals where $x_{1}$ and $x_{2}$ are close to 0 are widely separated.

- Fig. 21. Same conditions than for Fig. 20 except the initial condition for $y_{1}$ which is now : $y_{1}(0)=0.4$.

Nothing is changed except the shift between the two curves which is smaller. 


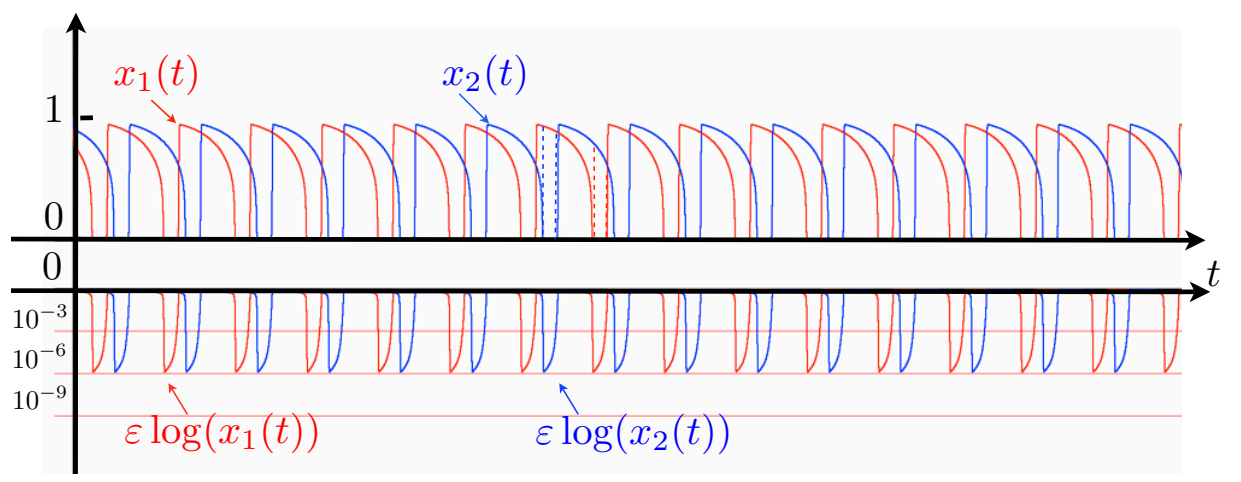

Figure 21. Model (10) $: \varepsilon=0.05 ; m=0.7 ; a_{x}=0.2 ; I=0.0001$. Initial conditions : $\left(x_{1}(0)=1 ; y_{1}(0)=0.4\right)$ and $\left(x_{2}(0)=1 ; y_{2}(0)=0.2\right)$

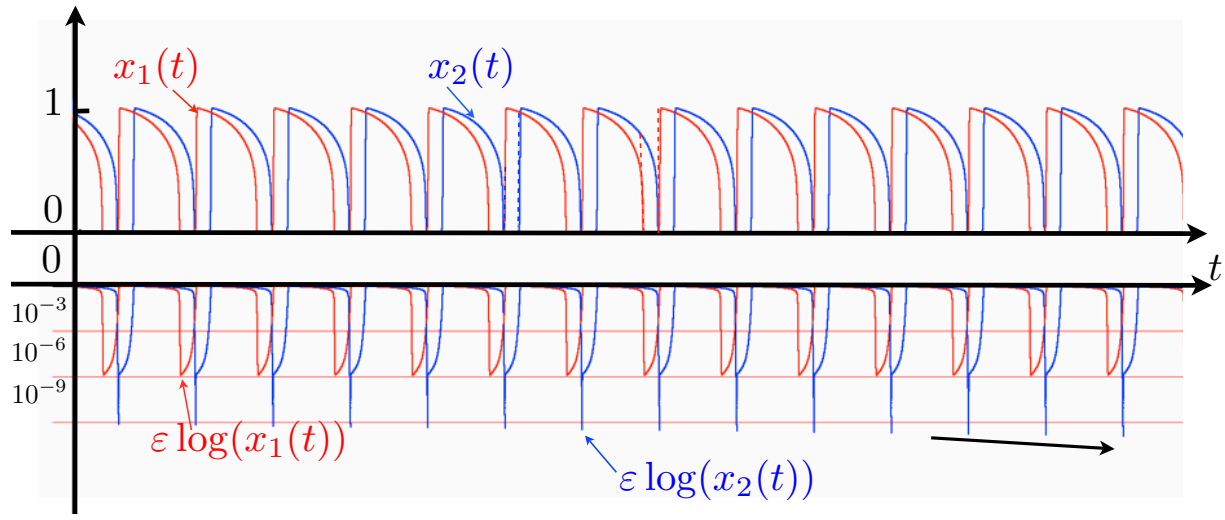

Figure 22. Model (10) $: \varepsilon=0.05 ; m=0.7 ; a_{x}=0.2 ; I=0.0001$. Initial conditions : $\left(x_{1}(0)=1 ; y_{1}(0)=0.31\right)$ and $\left(x_{2}(0)=1 ; y_{2}(0)=0.2\right)$

- Fig. 22. Same conditions than for Fig. 21 except the initial condition for $y_{1}$ which is now : $y_{1}(0)=0.31$.

There is a big change : the variable $x_{2}$, during a short period, is very small (less than $10^{-9}$ ) and by the way we have an"atto-fox problem". This is explained by the fact that now the shift is not sufficient; when the $x_{2}$ variable jumps to 0 the $x_{1}$ variable is just jumping from 0 and does not cross the threshold in time.

Moreover one sees that this minimum is decreasing in time which means that the jump of $x_{1}$ from 0 takes time later and later with respect to the jump of $x_{2}$ to 0 . In other words 


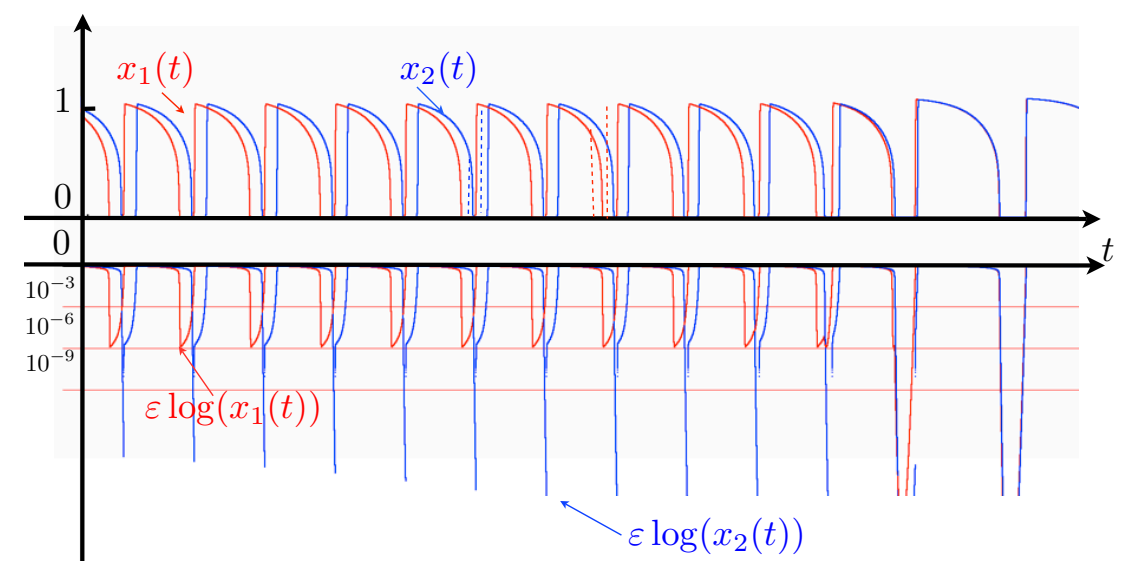

Figure 23. Model (10) $: \varepsilon=0.05 ; m=0.7 ; a_{x}=0.2 ; I=0.0001$. Initial conditions : $\left(x_{1}(0)=1 ; y_{1}(0)=0.3\right)$ and $\left(x_{2}(0)=1 ; y_{2}(0)=0.2\right)$

the difference of phases is decreasing.

- Fig. 23. Same conditions than for Fig. 22 except the initial condition for $y_{1}$ which is now : $y_{1}(0)=0.3$.

The phenomenon observed on the previous simulation is amplified ; at the end of the simulation the two sites are approximately synchronized and both have minima for $x$ which are very low.

\section{Conclusion}

The question of persistence is certainly a major issue in population dynamics. When the model is a system of differential equations the usual way to define uniform persistence (in the classical setting) is to say that there exists a strictly positive real $\alpha$ such that for any initial condition and any component $x_{i}$ of the system :

$$
\varliminf_{t \rightarrow \infty} x_{i}(t) \geq \alpha
$$

This is a first qualitative step but not sufficient. The usual Rosenzweig-MacAtrthur model with its globally asymptotically stable limit cycle is certainly persistent in that sense but, due to the "atto-fox" problem, might be not "persistent" as a model of an actual population. In our opinion, the language of NSA, with the possibility to ask the question whether the $\alpha$ in the above definition is infinitesimal or not, is particularly suitable to address this question. The analysis of the system in the variables $(\xi, y)$ gives explicit and accurate approximation of $\alpha$ for a small $\varepsilon$. 
In the literature the Rosenzweig-MacAtrthur model has been sometimes considered with "small" $\varepsilon$. It is for instance the case in the papers $[17,18,19]^{7}$ but, to our knowledge, the associated "atto-fox" question was never considered before us (see [16]). As we said in the introduction the Arditi-Ginzburg ratio-dependent model (see [1, 2]) is a better model than the Rosenzweig-MacArthur model. It differs from (3) by the fact the $y$-isocline is a line through the origin instead of being a vertical line ; this does not change the existence of a "jump" from the summit of the parabola to the vertical axis, "jump" which is responsible for the very small values of $x$ and, by the way, our present discussion applies to it.

In section 2.3 we have introduced a constant rate of immigration of the resource $I$ which prevents the extinction of the resource. There is another direction of research that we have not considered here : replace the deterministic differential system (3) by some suitable birth-and-death process like it was done in [7]. But in [7] the evaluation of the probability of extinction was not considered and this remains to be done.

If the existence of a constant immigration rate solves the "atto-fox" problem it supposes an external "continent" where the resource is always present. What about the situation for an isolated system ? In section 3.3 we have shown how a small nonlinear migration can prevent some solutions of a two patches system from an "atto fox" problem, at least for limited durations. It would have been better to prove it "forever" but we did not. We took advantage of the NSA language wich allows to say : this property is true for all limited $t$; this is not "for ever" but for a "long time".

In any case the present study is a very first step. It remains to consider various forms of migrations including migration of the consumer and more than two patches.

\section{Acknowledgements}

This paper was partly written while the authors where invited by the Centre Interfacultaire Bernoulli at the Ecole Polytechnique Fédérale de Lausanne in Switzerland.

The authors thank an anonymous referee. His (her) remarks allowed to improve considerably the paper. In particular we thank him (her) for pointing an unlimited number of missprints.

\section{References}

[1] ARditi R., L. R. GinzbuRg "Coupling in Predator-Prey Dynamics : Ratio-Dependency" Journal of theoretical biology vol. 139, 311-326 1989.

[2] ARditi R., L. R. GinzburG “How species interact”. Oxford University Press 2012.

7. Notice that the delay of the re-appearance of the resource was not known in the first two papers : see Fig. 2, 4, 7 in [17] and Fig. 2 in [18] which are false ; this mistake was corrected by the same authors in [19] (see Fig. 2). This phenomenon was already known as the "bifurcation delay" (see the collective book edited by E. Benoît [6]) but rediscovered independently in [19]) . 
[3] Benoît E. , J-L Callot , F. Diener , M. Diener. "Chasse au canard" Collectanea Mathematica, vol. 31-32 (1-3) p. 37-119 1981.

[4] BENOÎT E. Site personnel. http://perso.univ-lr.fr/eBenoit/.

[5] E. BEnOÎT "Tunnels et entonnoirs", Comptes-Rendus de l'Académie des Sciences de Paris, série I, vol. 292 : p. 283-286, 1981.

[6] Benoît E. (ED) "Dynamic Bifurcations : Proceedings, Luminy 1990". Lecture Notes in Mathematics vol. 1493 Springer-Verlag1991.

[7] CAmpillo F. , C. LOBRY "Effect of population size in a predator-prey model" Ecological Modelling vol. 246, p. 1-10 2012.

[8] CAMpillo F. , C. LOBRY "Effet de la migration dans la relation ressource-consommateur du point de vue de "l'atto-fox problem"” HAL INRIA,hal-00800370 2013.

[9] Cheng K. "Uniqueness of a limit cycle for a predator-prey system" SIAM J. Math. Anal. vol. 12, p. 541-548 1981.

[10] Diener F. , M. Diener.(Eds). "Nonstandard Analysis in Practice" Springer (Univesitext) 1995.

[11] GouzÉ "Positivity, space scale and convergence toward the equilibrium" J. Biol. Syst. vol. 03 p. 613-620 1995.

[12] Holt R. "Population Dynamics in Two-Patch Environments : Some Anomalous consequences of an Optimal Habitat Distribution" Theoretical Population Biology vol. 28. num. 2 , p. 181-208 1995.

[13] V. JANSEN “The Dynamics of Two Diffusively Coupled Predator-Prey Populations” Theoretical Population Biology vol. 59, p. 119-1312001.

[14] LOBRy C. "Sur le sens des textes mathématiques, un exemple : la théorie des "bifurcations dynamiques"” Annales de l'Institut Fourier, vol. 42, p.327-351 1992.

[15] LOBRy C. , T. SARI. "Nonstandard analysis and representation of real world" International Journal on Control, vol. 80(3) p. 171-193 2007.

[16] LOBRY C. , T. SARI. "La modélisation de la persistance en écologie" Colloque à la mémoire d'Émmanuel Isambert, Université Paris 7, Dec 2007, Paris, France. Publications de l'Université Paris 13, pp.163 - 184. <hal-00824769>, 2007.

[16] Mollison D. "Dependence of epidemic and population velocities on basic parameters" Math Biosciences, vol. 107, p.255-287 1991.

[17] Muratori S., S. Rinaldi. "Remarks on competitive coexistence" SIAM J. Appl. Math., vol. 49, p. 1462-1472 (1989).

[18] Muratori S., S. Rinaldi. "A separation condition for the existence of limit cycles in slowfast systems” Appl. Math. Mod., 15, p. 312-318 1991.

[19] Rinaldi S., MURATORI S. "Slow-fast limit cycles in predator-prey models" Ecological Modelling vol. 61, p. 287-308 1992.

[20] Nelson E. "Internal Set Theory” Bull. Amer. Math. Soc., vol. 83, p. 1165-1198 1977.

[21] REDER C. "Familles de convexes invariantes et équations de diffusion réaction" Annales de l'Institut Fourier, vol. 32, p. 71-103, 1982. 